\title{
Responding to the Antisocial Personality Disorder and Narcissistic Personality Disorder: Etiology, Challenges, and Treatment
}

\author{
Mutian $\mathrm{Pi}^{1, \mathrm{a}, \dagger} \mathrm{Yu} \mathrm{Zuo}^{2, \mathrm{~b}, \dagger}$ \\ ${ }^{1}$ Beijing National Day School, Beijing, 100039, China \\ ${ }^{2}$ Beijing No.4 High School, Beijing, 100031, China

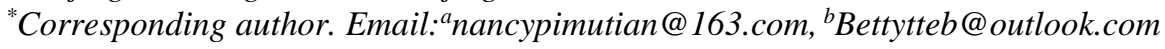 \\ These authors contributed equally.
}

\begin{abstract}
Personality disorder: the set of enduring behavioral and mental traits that distinguish an individual from other people. Based on the DSM-5, ten different personality disorders are grouped into three clusters based on common features cluster A, cluster B, and cluster $\mathrm{C}$. The paper focuses on cluster B, which is characterized by personality styles that are impulsive, dramatic, highly emotional, and erratic. Cluster B disorders include antisocial personality disorder, histrionic personality disorder, narcissistic personality disorder, and borderline personality disorder. The paper mainly discusses antisocial and narcissistic personality disorder. A person with antisocial personality disorder continuously violates the rights of others; often lies, fights, and has problems with the law; he or she can be deceitful and manipulative to gain profit or pleasure, and the patient lacks feelings for others and remorse over misdeeds. And people with narcissistic personality disorder have an overinflated and unjustified sense of self-importance and are preoccupied with fantasies of success. They believe they are entitled to special treatment from others, take advantage of others, and lack empathy. Case studies involved in the following paper will give authentic examples to demonstrate the specific symptoms of those disorders. Based on the features of being hard to communicate with, lacking remorse, tending to be violent, and being difficult to realize that they need psychological or medical help, patients with cluster B disorders are difficult to be treated. Although some treatments are developed and used to treat those disorders, few of them are effective and qualified. Hence, the paper contains studies and experiments that aim to question and qualify the effect and efficiency of specific treatment of Cluster B disorders. The future development of the treatment of Cluster B disorders is also expected in the last part of the paper.
\end{abstract}

Keywords: Personality disorder, Antisocial Personality Disorder, Narcissistic Personality Disorder, Etiology, Obstacles, Treatment, Case Study, Cognitive Behavioral Therapy,

\section{INRODUCTION}

The essay depends mainly on the research papers found on the Internet and the definition of psychological concepts on an authoritative website. For different parts, different case studies and experiments are discussed in the next part of the essay. Those studies and experiments are from sources found through the Internet.

\subsection{About ASPD}

The methodology used for this research paper is a review of pertinent literature relying on articles of studies. A literature search was conducted using Google Scholar to collect the general information of basic information of ASPD and studies related to ASPD treatment. The sources in this paper are articles from reliable sources. The primary sources are the article "How to Treat Antisocial Personality Disorder" and an article from the book Psychological Medicine which discusses the study on Cognitive Behavior Therapy for people with ASPD. The latter article focuses on the effectiveness and the feasibility of using CBT to treat men with APSD who were aggressive. 


\subsection{About NPD}

The essay is mainly completed by searching for documents on the Internet. The article searches for keywords on the Internet: narcissistic personality disorder, pathology, and learns the general content of NPD from lumen learning. Finish this paper by sorting out the main content in the literature. The primary resource is Narcissistic Personality Disorder: Progress in Recognition and Treatment from Psychiatry Online.

\section{DISCUSSION AND RESULT}

\subsection{ETIOLOGY}

Before discussing treatments, understanding the etiology of two personality disorders are essential. A combination of genetic and environmental influences leads to personality disorder. Genetics is usually proposed by scientists as a prior biological character in most psychological disorders. ASPD has some or even a strong genetic basis. Twins studies have shown that heredity plays a role in antisocial behavior. Specifically, the gene that codes for Monoamine oxidase $\mathrm{A}$ is correlated to ASPD [1]. Vary studies show that less Monoamine Oxidase A results in aggressive behavior in men. Also, the gene encodes for the serotonin transporter plays a role [2]. In addition, ASPD is often caused by generating hormones such as testosterone and serotonin. A study shows that testosterone is a hormone that is higher in patients associated with ASPD [3]. Head trauma leads to the change of the grey matter in the right lentiform nucleus, left insula, and frontopolar cortex. Meanwhile, scientists have not discovered a clear genetic cause of Narcissistic Personality Disorder yet. Although the causes of NPD are still vague, some speculations are given for psychiatrists to research. Study shows that a molecule named 8-hydroxy-2'-deoxyguanosine(8-OHDG), an oxidative stress biomarker is highly related with narcissistic personality disorder [4]. Psychologists researched about a hundred and ninety-five people. It was letting them do a questionnaire to estimate their cognition and emotion. Then they took the blood sample from the subjects. The result appears that people with NPD showed a distinctive level of 8-OH-DG. Furthermore, this study demonstrated the effect of 8-OH-DG level on narcissistic personality disorder by linear regression line model, independent of age, gender, the traumatic event experienced, and other confounding variables. Broadline personality disorder, as a disorder that shares one hand feature with narcissistic personality disorder, is also identified as associated with the impact of 8-OH-DG level. Scholars further believe that the 8-OH-DG level may not directly lead to the generation of cluster B personality disorder but is related to the attribution of Disgust. Because the experiment still has space to improve (e.g., small sample), the experimental results still have uncertain factors.
A combination of genetic and environmental influences leads to personality disorder.

For environmental factors, the family environment plays an important role in children's development. A lack of parental stimulation and affection leads to the inability of children to deal with emotion, empathy, and connection with other people on an emotional level [5]. If a child was suffered from physical or sexual abuse, he or she is likely to develop an ambivalent attachment and low self-esteem. They could build their attachment by forced themselves to be perfect. Scientists have the same inferences about the early relationships between patients and their friends. But there is still little reliable data on which to base it, and it is not clear which specific types of environmental factors are highly associated with NPD

Different cultures view ASPD differently, which makes cultural influences become a cause of ASPD. However, there are still lots of debates related to the cultural impacts. Moreover, prisoners and people in alcohol or other drug abuse treatment programs have a higher risk of having ASPD; ASPD is associated with gender that ASPD is diagnosed more frequently in men than women.

Finally, the natural personality and the hypersensitivity to textures, noise, or light in childhood are also considered by scientists.

\subsection{RELATED IMPACTS}

Having cluster B disorders will suffer from negative impacts on individuals and have negative consequences on patients' families, friends, and the society they live in.

First, researches show that ASPD and NPD can be related to comorbidities. ASPD can be related to anxiety disorder, Bipolar disorder, Post-traumatic disorder, Attention deficit hyperactivity disorder, borderline personality disorder, and schizotypal personality disorder [6]. NPD Patients forced themselves to be attractive, successful and craved praise and admiration from other people. However, behind their ignorant, vulnerable selfesteem, compulsive thoughts always bring them comorbidity. While NPD can be related to depression, mania, and so on combined with their personality disorder, bringing pain for their life. The most common comorbidity in major depressive disorders. Even if the symptoms of NPD are a bit contradicted to the symptoms of major depressive disorders, according to the research, about $45 \%$ to $50 \%$ who suffered from NPD have also received the treatment of depression.

The case study identifies the major symptoms of narcissistic personality disorder. The paper focused on and necessarily offering a better way to understand it. In a case study, Mr. V with NPD claimed that he had lost interest in most things because he could not tolerate the stupidity of them. He was afraid to communicate with 
people because they might do things behind his back that are detrimental to his reputation. His work productivity was extremely low; He suggested that people's disapproval of his work was all because of jealousy. Thus, criticism from others would lead to his anxiety through his external locus would not let him take the responsibility on his own. Mr. V is married, but he was sexually inactive. He thought these behaviors are stupid things that people with empty heads would do. As a result, Mr. V was diagnosed with major depressive disorder and anxiety disorder.

Though those disorders can be treated with psychotherapy and medication, since patients with ASPD and NPD tend to refuse to seek help, it is more difficult for them to be recovered from mental diseases. People with personality disorders are not a minority. According to statistics, one out of hundred randomly selected people has NPD. The data indicates that there is an urgent need for the treatment of personality disorders.

And a study shows that ASPD can lead to smoke, use illegal drugs, and be more dependent on alcohol [7]. Physical health is threatened for ASPD patients because they tend to make choices without considering personal safety.

Based on the symptoms of less likely to seek, talk, and establish close relationships with others, people with ASPD or NPD are more likely to have problems maintaining healthy relationships and holding down jobs, which in turn deeply affects the ordinary life of them. Mr. $\mathrm{D}$ was an administrative assistant in the family business. He demanded the best for himself. The women he had dated could not disagree about his family or him, or they will get dismissed. The upset ending about a relationship with an NPS patient is likely to happen.

Finally, the higher cost of these two personality disorders is the impacts it brings to the society. As for ASPD, violent and criminal behavior will happen to them. The security and safety of patients, clinicians, counselors, therapists, and even ordinary people are threatened in that case. Severely, victims' physical and mental health condition being attacked by people with ASPD will be harmed heavily. For an individual who's in a relationship with an NPD patient, one may be received physical abuse, verbal insults, and mental suffering from the relationship.

\subsection{GENERAL TREATMENT}

Due to the severe impacts ASPD and NPD bring to individuals and societies, their treatments are important and crucial. However, ASPD is considered to be a difficult personality disorder to be treated. Kornberg also acknowledges NPD patients that some are "almost impossible to cure." Because of their low or absent capacity for remorse, patients lack motivation to change themselves and see themselves as being sick.
ASPD may be treated with residential programs that offer a controlled environment of structure and supervision along with peer confrontation. Psychotherapy can be used as well. However, for patients with severe symptoms, psychotherapy will be ineffective. Schema therapy and Multisystemic therapy are also evaluated to be used to treat ASPD. Unfortunately, they still need to develop and improve. The use of medication in treating ASPD still needs to be explored. Cognitivebehavioral therapy (CBT) is being tested in the study done by K. M. Davidson, P. Tyrer, P. Tata, D. Cooke, A. Gumley, I. Ford, A. Walker, V. Bezlyak, H. Seivewright, H. Robertson, and M. J. Crawford[8].

As for NPD, There is no single strategy that has been recognized by psychologists, although long-term therapy does have always been the primary treatment for NPD clients as they do not have any serious biological symptoms. NPD can be treated at different stages for a variety of reasons. It is necessary to address the initial contact with a narcissistic patient to encourage them to explore relevant issues and encourage them to be willing to address these issues in a meaningful way. It is especially important to determine the patient's understanding and description of the problem and the motivation to seek treatment, which may take several sessions to reach such an agreement. Therapists use a flexible approach, tailored to the individual's level of pathology, motivation, self-awareness, and a respectful, consistent, focused, and patient-centered approach. There are plenty of factors that may influence the cognition of Therapist mainly working on changing five domains essential for the treatment of NPD clients: Interpersonal and vocational functioning, sense of agency, emotion regulation, reflective ability, ability to mourn.

\subsection{INTRODUCTION TO COGNITIVE BEHAVIOR THERAPY}

Cognitive Behaviour Therapy aims to improve mental health. CBT focuses on challenging and changing cognitive distortions and behaviours, improving emotional regulation, and developing personal coping strategies targeting current problems. The role of therapists in using CBT is to help patients finding effective methods to decrease symptoms of the disorder. A typical CBT program consists of face-to-face sessions between therapists and patients. CBT can be seen as having six phases based on the system created by Kanfer and Saslow. CBT is usually to treat depression, anxiety disorder, bipolar disorder, etc. The techniques used for treating ASPD are not mature enough. The study conducted in the article "Cognitive behavior therapy for violent men with antisocial personality disorder in the community: an exploratory randomized controlled trial" ends up the result that the study ends up with the result of "CBT for men with ASPD who were aggressive had no significant effect compared with usual treatment on 
anxiety, depression, anger, harmful drinking, negative or positive beliefs about self or others and social functioning at 12 months of follow-up" and "We noted trends, however, for those who received CBT to report more positive beliefs about others, and for less harmful alcohol use compared to TAU. The latter effect on harmful drinking was also confirmed in the 6-months CBT group." The result shows that CBT does make a difference in changing unhealthy life habits such as depending on alcohol, using drugs, and smoking; however, how to solve the problem with ASPD completely is still a question that waits for answers.

\section{CONCLUSION}

In conclusion, the research paper discusses the etiology, impacts, general treatments of antisocial personality disorder and narcissistic personality disorder. In etiology, genetic and environmental factors are all related to the happening of ASPD and NPD. In impacts, ASPD and NPD both bring negative impacts to patients themselves and society. ASPD and NPD still require effective and high-quality methods in general treatment since modern techniques are not universally efficient. For CBT, although it can make a difference in changing the surface symptoms of some patients, it is difficult for it to solve the problem from the basis. The treatments of narcissistic personality disorder and antisocial personality disorder still have many difficulties to overcome. The complex composition of personality disorders and the lack of successful, well-developed Census surveys are big obstacles. In the future, psychologists will continue to search for effective and targeted treatments for both personality disorders. While people started to know more about psychopathology, ASPD and NPD are still tending to be neglected as a psychological disorder. Thus, people with NPD and ASPD do suffer a lot. Not only to the negative symptoms that ASPD and NPD have brought, but also criticisms from most people who do not understand what ASPD and NPD are. Thus, we may treat people with different personalities with respect and open-mindedness.

\section{REFERENCES}

[1] Antisocial Personality Disorder, https://en.wikipedia.org/wiki/Antisocial_personality _disorder\#Treatment.

[2]Cognitive Behavioural Therapy, https://en.wikipedia.org/wiki/Cognitive_behavioral _therapy\#Phases_in_therapy

[3] Elsa Ronningstam and Igor Weinberg., Narcissistic Personality Disorder: Progress in Recognition and Treatment.Available at: https://focus.psychiatryonline.org/doi/full/10.1176/a ppi.focus.11.2.167
[Accessed in Apr 2013]

[4] Harry Croft., The Narcissistic Patient - A Case Study. Available at: https://www.healthyplace.com/personalitydisorders/malignant-self-love/narcissistic-patient-acase-study

[Accessed in July 5, 2018]

[5] V. Forejt, M. Kwiatkowska, G. Norman, D. Parker, Automated verification techniques for probabilistic systems, in: M. Bernardo, V. Issarny (Eds.), Proceedings of the Formal Methods for Eternal Networked Software Systems (SFM), Springer, Berlin, Heidelberg, 2011, pp. 53-113. DOI: https://doi.org/10.1007/978-3-642-21455-4_3

[6] G.D. Penna, B. Intrigila, I. Melatti, E. Tronci, M.V. Zilli, Bounded probabilistic model checking with the muralpha verifier, in: A.J. Hu, A.K. Martin (Eds.), Proceedings of the Formal Methods in ComputerAided Design, Springer, Berlin, Heidelberg, 2004, pp. 214-229. DOI: https://doi.org/10.1007/978-3540-30494-4_16

[7] E. Clarke, O. Grumberg, S. Jha, et al., Counterexample-guided abstraction refinement, in: E.A. Emerson, A.P. Sistla (Eds.), Computer Aided Verification, Springer, Berlin, Heidelberg, 2000, pp. 154-169.

DOI: https://doi.org/10.1007/10722167_15

[8] H. Barringer, R. Kuiper, A. Pnueli, Now you may compose temporal logic specifications, in: Proceedings of the Sixteenth Annual ACM Symposium on the Theory of Computing (STOC), ACM, 1984, pp. 51-63. DOI: https://doi.org/10.1145/800057.808665 